\title{
Modified Rib Sparing Direct Lateral Minimally Invasive Vertebrectomy for Treatment of Metastatic Spinal Cord Compression
}

\author{
Mark Nowell, Benedict Hughes, Peter Loughenbury, Jake Timothy \\ Leeds General Infirmary, Leeds, UK
}

Corresponding Author:

Mark Nowell, PhD, FRCS

Spine Fellow, Leeds General

Infirmary, Leeds, UK

Tel: +44-7812997874

E-mail: mnowell@gmail.com

Received: November 17, 2018

Revised: March 28, 2019

Accepted: May 15, 2019

\begin{abstract}
Objective: Metastatic spinal cord compression (MSCC) is a common complication of metastatic disease with neurological morbidity in the thoracic and upper lumbar spine. We describe a modified rib-sparing direct lateral minimally invasive (MIS) approach. for the purpose of vertebrectomy. This technique obviates the need for rib resection and chest drain, facilitating early mobilisation. This is especially applicable to a sub-group of patients who may not be well enough for an open approach. Method: Technical note and retrospective case series in a single centre over a 5 year period. Results: 14 patients were identified who underwent the MIS approach vertebrectomy for MSCC. 12/14 underwent posterior fixation, and 2 underwent vertebroplasty. 11/14 (79\%) had less than $1 \mathrm{~L}$ blood loss during the procedure. The mean duration of the procedure was 5 hours 51 minutes. 5/14 (36\%) avoided high dependency unit (HDU) care, and the median duration of time spent in HDU was 1.5 days. The median length of stay in hospital was 16 days, and 4/14 (29\%) were discharged within 1 week. There were 1/14 major complications requiring revision surgery. Conclusion: A modified rib-sparing MIS approach for vertebrectomy is well tolerated in the treatment of MSCC and is associated with low blood loss and short hospital stays. This may be an option in patients who otherwise may not be considered for an anterior reconstruction.
\end{abstract}

Key Words: Metastatic spinal cord compression, Vertebrectomy, XLIF

\section{INTRODUCTION}

Metastatic spinal cord compression (MSCC) is a common complication of metastatic disease, with high neurological morbidity ${ }^{2}$. Surgical decompression improves outcomes in selected patients, by preserving walking and sphincter function ${ }^{4}$. Anterior column decompression and reconstruction, supplemented with posterior fixation is the gold standard surgical treatment of MSCC when the tumour arises from the vertebral body. Surgical access to the anterior column has conventionally been through open lateral approaches. Open surgery is associated with pulmonary complications and may only be tolerated by patients who have better overall health scores.

The transpsoas extreme lateral interbody fusion (XLIF) approach is a minimally invasive procedure first described to gain access to the anterior column of the lumbar spine for the purposes of discectomy and interbody cage fusion ${ }^{3}$. This technique has been modified to allow for more extensive access across the thoracolumbar spine, and for addressing other pathologies affecting the anterior column ${ }^{1)}$. The main advantage of this app- roach is that it is better tolerated by the patients, with fewer pulmonary complications.

We describe our 5-year experience with the surgical treatment of MSCC arising from the anterior column where vertebrectomy was indicated. Patients were managed with a modified direct lateral MIS approach procedure.

\section{TECHNICAL NOTE}

Double lumen ventilation is not required and the patient is ventilated normally throughout the majority of the procedure. This provides an immediate advantage over the open procedure where one lung is routinely collapsed. The patient is placed in the lateral position. A small incision is made over the rib overlying the vertebra of interest, determined by lateral image intensifier imaging (Fig. 1A, (B)). The plane between the superior surface of the rib and the intercostal muscles is developed both anteriorly and posteriorly by blunt dissection using the index finger alone, to allow for undermining and rib mobilisation (Fig. 1(C), (D). Momentum of the finger is necessary to strip the muscle off the superior aspect of the rib. Dissection with the 

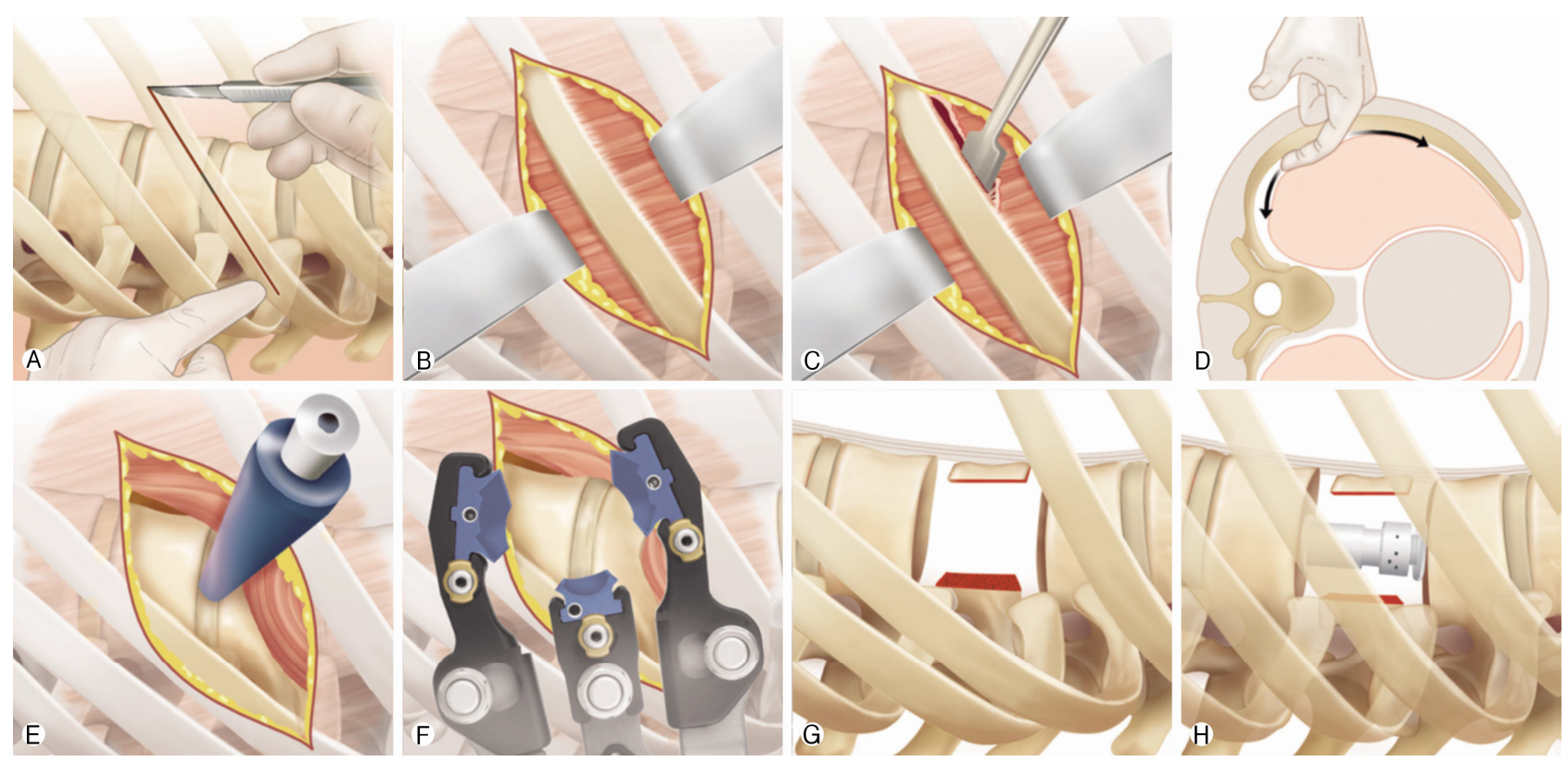

Fig. 1. Illustration of principle stages of minimally invasive modified direct lateral transthoracic approach (MIS). (A) Incision over rib of interest with patient in true lateral position, (B) Exposure of rib, (C) Development of plane between superior aspect of rib and intercostal muscles, (D) Blunt dissection behind rib ventrally and dorsally in retropleural space with preservation of pleural membranes, (E) Placement of guidewire and dilators over rib space with image intensifier guidance, ( $)$ Placement and opening of retractors to gain exposure to disc space of interest, (G) Corpectomy, $\mathbb{A}$ Cage placement.

finger is taken under the skin incision to as anterior as possible to the transverse process of the vertebra of interest, with care taken to minimise/avoid incursion through the pleura although in most cases the pleura is breeched (Fig. 1(D). If so, the ventilation is ceased and the lung is retracted with a Penfield or fingers and protected away from the anterior vertebral column and discs. A guidewire and dilators are placed between the ribs, under image intensifier with a temporary cessation of ventilation that typically lasts 2-3 minutes (Fig. 1(E), (F). The retractors are then slid over the dilators and are opened very gradually to allow rib displacement out of the surgical field. Lung tissue is protected by shims inserted within the retractor set to enable full ventilation. At the thoracolumbar junction the diaphragm insertions can be diathermied off the vertebral body. In the lumbar spine the retractors are placed transpsoas, and we recommend a primary 'soft dock' superficial to psoas to allow for direct visualisation and transposition of the nerves of the lumbosacral plexus.

Corpectomy and cage placement are then performed in the standard manner (Fig. 1(G), $(\mathbb{H})$.

At the end of the procedure the intercostal muscles overlying the rib are closed, with care taken to avoid injury to the neurovascular bundle underlying the rib. A purse string suture is placed and the anaesthetist induces hyperinflation in the patient prior to final closure to remove excess air from any pleural cavity defects. The patient requires close observation and chest radiography in recovery to ensure no residual large pneumothorax. No patients in this group needed an emergency placement of a chest drain in the postoperative period.
In our experience (JT) the advantage of this technique is that it is better tolerated by the patient. Firstly, there is optimal ventilation intraoperatively. Secondly there are smaller incisions with sparing of the rib and avoidance of chest drains, that contributes to earlier mobilisation and less post-operative pain.

\section{MATERIALS AND METHODS}

14 patients underwent this procedure from August 2012August 2017.

Demographic and outcome data for selected patients was found from their electronic records on the Patent Pathway Manager (PPM), that included initial surgical assessment, dates of ward discharge and surgical and oncological follow up. The operation notes were found on the PPM in the format of a typed document (Bluespier). Surgical times were recorded on the Galaxy theatre operations system. The original clinical notes were pulled to provide further supporting information.

For each patient we identified demographic information, primary tumour histology, and surgery type including levels of surgery and supplementation with posterior decompression/fixation. Primary outcomes were estimated blood loss determined from operation note, duration of surgery, length of stay in high dependency unit and length of stay in the hospital. Secondary outcomes were procedure-related complications.

The patients were primarily operated by a single surgeon (JT), using a variety of lateral access retractors, including Nuvasive, Globus Medical and RTI Surgical products. 

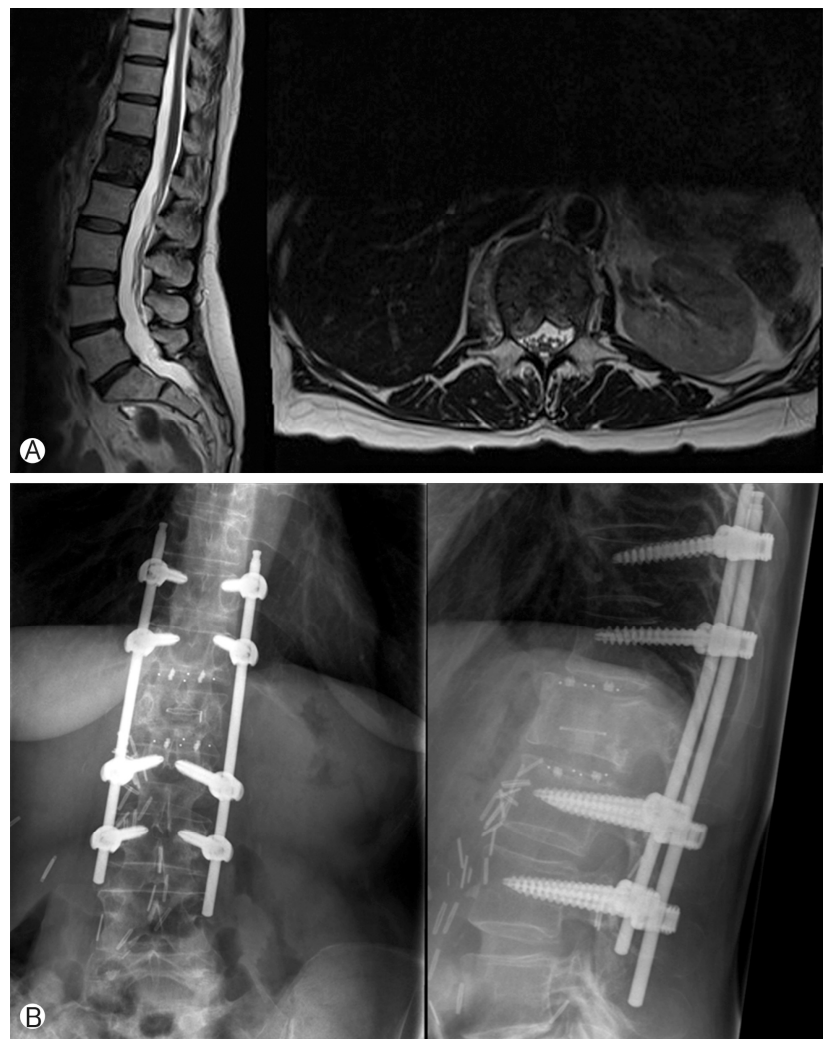

Fig. 2. Pre and post operative imaging for patient 5. (A) Pre-op T2 weighted mid-sagittal MRI and axial of lumbar spine showing metastatic invasion of $L 1$ vertebral body extending into pedicles with no deformity or neural compression. (B) Post operative standing $\mathrm{X}$-rays in lateral and anteroposterior position showing L1 corpectomy cage and MIS instrumented fixation from T11-L3.

\section{RESULTS}

14 patients were identified undergoing the MIS approach. The demographic details and operations are listed in Table 1.

An example of the surgery is demonstrated in Figure 2 for patient 5 .

10/14 patients underwent supplemental posterior fixation. These were a combination of open and percutaneous, with additional posterior decompressions in some cases where tumour was causing posterior compression as well. This was performed through a small midline laminectomy approach centred between the percutaneous pedicle screw fixation which was either one or two levels above and below the infiltrated vertebra.

The outcomes of the patients are shown in Table 2. 11/14 (79\%) had less than $1 \mathrm{~L}$ blood loss during the procedure. The mean duration of the procedure was 5 hours 51 minutes. 5/14 $(36 \%)$ avoided HDU, and the median duration of time spent in HDU was 1.5 days. 3 patients required a chest drain, but none as an emergency. The median length of stay in hospital was 16 days, and 4/14 (29\%) were discharged within 1 week. There was 1 major complication requiring revision surgery although this was unrelated to the direct lateral approach. Patient 3 deteriorated neurologically post operatively due to an epidural haematoma that was evacuated. At the same time a pedicle screw position was modified for optimisation.

\section{DISCUSSION}

Surgical treatment of MSCC is challenging and high risk. Robust referral pathways are required locally to ensure rapid diagnosis and appropriate onwards referral with input from on-

Table 1. Table to show individual patients demographics and operation type in case series

\begin{tabular}{|c|c|c|c|c|c|c|}
\hline & Age & Gender & Pathology & Vertebrectomy level & Posterior fixation & Additional procedures \\
\hline 1 & 59 & $M$ & Teratoma & $\mathrm{L} 2$ & Y (open) & \\
\hline 2 & 56 & M & Lung & L2 & Y (open) & Posterior decompression \\
\hline 3 & 73 & M & Bladder & L3 & Y (open) & Posterior decompression \\
\hline 4 & 60 & M & Prostate & T8 & $\mathrm{N}$ & Vertebroplasty \\
\hline 5 & 55 & $\mathrm{~F}$ & Renal cell & L1 & Y (MIS) & \\
\hline 6 & 72 & $M$ & Caecal & L3 & Y (MIS) & \\
\hline 7 & 48 & $\mathrm{~F}$ & Breast & L1 & $\mathrm{N}$ & Vertebroplasty \\
\hline 8 & 65 & $\mathrm{~F}$ & Renal & L1 & Y (MIS) & \\
\hline 9 & 68 & $\mathrm{~F}$ & Breast & $\mathrm{T} 12$ & $\mathrm{~N}$ & \\
\hline 10 & 67 & M & Tonsillar & T9 & Y (MIS) & \\
\hline 11 & 76 & M & Renal cell & $\mathrm{T} 12$ & Y (open) & Posterior decompression \\
\hline 12 & 37 & M & Myeloma & L4 & Y (open) & Posterior decompression \\
\hline 13 & 82 & $M$ & Colon & L2 & Y (open) & Posterior decompression \\
\hline 14 & 67 & M & Myeloma & $\mathrm{T} 7$ & $\mathrm{~N}$ & \\
\hline
\end{tabular}

Y: yes; N: no; $M$ : male; F: female. 
Mark Nowell, et al.

Table 2. Table to show individual patient outcomes from surgery

\begin{tabular}{|c|c|c|c|c|c|c|c|}
\hline Patient & $\begin{array}{l}\text { Blood loss } \\
(\mathrm{mL})\end{array}$ & $\begin{array}{l}\text { Duration } \\
\text { (hrs) }\end{array}$ & $\begin{array}{l}\text { LOS HDU } \\
\text { (days) }\end{array}$ & $\begin{array}{c}\text { Total LOS } \\
\text { (days) }\end{array}$ & Chest drain & Complications & $\begin{array}{l}\text { Survival } \\
\text { (months) }\end{array}$ \\
\hline 1 & $100-200$ & 5:01 & 0 & 5 & $\mathrm{~N}$ & Nil & 6 \\
\hline 2 & $500-1,000$ & $4: 13$ & 1 & 15 & $\mathrm{~N}$ & Nil & 1 \\
\hline 3 & $>1,000$ & $6: 17$ & 5 & 50 & $\mathrm{~N}$ & $\begin{array}{l}\text { Revision surgery for epidural haematoma } \\
\text { evacuation and pedicle screw revision }\end{array}$ & 20 \\
\hline 4 & $100-200$ & $4: 56$ & 7 & 13 & $\mathrm{~N}$ & Cellulitis, URTI, Mild pulmonary oedema & 10 \\
\hline 5 & $>1,000$ & $7: 47$ & 6 & 11 & $\mathrm{~N}$ & Nil & 33 \\
\hline 6 & $200-500$ & $5: 57$ & 3 & 15 & Y (post op) & Confusion, pneumothorax & 1 \\
\hline 7 & $200-500$ & $5: 25$ & 1 & 10 & $\mathrm{~N}$ & Nil & Alive (30) \\
\hline 8 & $200-500$ & $6: 26$ & 0 & 4 & $\mathrm{~N}$ & Nil & 7 \\
\hline 9 & 200 & $5: 38$ & 0 & 4 & $\mathrm{~N}$ & Nil & Alive (28) \\
\hline 10 & $100-200$ & $4: 22$ & 2 & 18 & Y (post op) & Pneumothorax & 11 \\
\hline 11 & $500-1,000$ & $8: 44$ & 7 & 15 & $\mathrm{~N}$ & Nil & Alive (18) \\
\hline 12 & $500-1,000$ & $5: 33$ & 0 & 9 & NA & Nil & Alive (6) \\
\hline 13 & $100-200$ & $U$ & 0 & 5 & $\mathrm{~N}$ & Nil & 4 \\
\hline 14 & $>1,000$ & $6: 30$ & 9 & 54 & Y & Pre op LRTI, pulmonary oedema & 9 \\
\hline
\end{tabular}

LOS: length of stay; HDU: high dependency unit; U: unknown; NA: not applicable; LRTI: lower respiratory tract infection; URTI: upper respiratory tract infection.

cologists and spine surgeons, within a multi-disciplinary framework if possible as per NICE guidelines ${ }^{5}$. This clinical problem is increasing in frequency with an ageing population, better access to imaging and new oncological treatments. Surgical treatment remains the gold standard, and the advent of minimally invasive techniques opens the potential to provide surgical treatment to a wider group of patients.

We describe a novel technique of a rib sparing minimally invasive direct lateral approach to the thoracolumbar spine. This case series demonstrates that this approach is feasible and safe. There is typically a high risk of complications with these patients, reflecting the nature of the surgery and the underlying pathologies. In this case series there was only 1 major complication requiring revision surgery. There were 2 patients that required insertion of chest drains in the post-operative period, which was done in a controlled manner with no harm to the patients. We contend that patients do not require chest drains as standard, although they do require immediate post-operative chest radiography and close observation in the first 24 hours for evidence of respiratory compromise. All patients planned for the minimally invasive approach completed their vertebrectomy without removal of rib or formal thoracotomy. The only situation where we would anticipate difficulties with the rib sparing MIS approach would be where there is reduced chest compliance limiting rib distraction, such as patients with ankylosing spondylitis.

In summary we contend that minimally invasive vertebrectomy is feasible and safe, and potentially offers patients lower 'impact' surgical treatment. This can be applied to all pathologies including infection, trauma and degenerative disease. However, it is most applicable and beneficial in treating MSCC where patients are often frail with multi-organ involvement and poor physiological reserve. As oncological treatments improve and surgery becomes better tolerated, there is a case for a rethink of the role of surgery, which historically has been restricted to preserving neurological function in patients with reasonable oncological prognoses. In this series there are patients with very poor prognoses, where the role of surgery was to treat unremitting mechanical pain refractory to medical management rather than preservation of neurological function. As oncological medical treatments improve and evolve, there should be a corollary innovation in the role of surgical treatment and the form that it takes.

\section{CONCLUSION}

MIS vertebrectomy is feasible and safe, and potentially offers patients low 'impact' surgical treatment for MSCC, broadening the patient population who are appropriate for this surgery.

\section{ACKNOWLEDGEMENTS}

We would like thank Globus Medical, Inc. (Audubon, PA) for their assistance in providing the medical illustrations used in this manuscript.

\section{Disclosures}

Funding: There has been no funding for this research. 
Conflict of Interest: Peter Loughenbury and Jake Timothy have received speaker honorariums from Stryker Education. Jake Timothy has received speaker honorariums from Baxter, Globus Medical and RTI Surgical.

Portions of this work were presented in a podium presentation at Britspine, Leeds, March 2018.

\section{REFERENCES}

1. Meredith D, Kepler C, Huang R, Hegde V: Extreme lateral interbody fusion (XLIF) in the thoracic and thoracolumbar spine: Technical Report and Early Outcomes. HSS Journal 9 (1):25-31, 2013
2. Nater A, Sahgal A, Fehlings M: Management - spinal metastases. Handbook of Clinical Neurology 149:239-255, 2018

3. Ozgur B, Aryan H, Pimenta L, Taylor W: Extreme lateral interbody fusion (XLIF): a novel surgical technique for anterior lumbar interbody fusion. The Spine Journal 6(4):435-443, 2005

4. Patchell R, Tibbs P, Regine W, Payne R, Saris S, Kryscio R, Mohiuddin $\mathrm{M}$, Young B: Direct decompressive surgical resection in the treatment of spinal cord compression caused by metastatic cancer: a randomised trial. The Lancet 366:643-648, 2005

5. White B, Stirling A, Paterson E, Asquith-Coe K, Melder A: Diagnosis and management of patients at risk of or with metastatic spinal cord compression: summary of NICE guidance. BMJ (Clinical Research ed.) 337:a2538, 2008 